\title{
Design and Performance of Laser-Pumped Cs-Magnetometers for the Planned UCN EDM Experiment at PSI
}

\section{Volume 110 \\ S. Groeger \\ Université de Fribourg, Chemin de Musée 3, 1700 Fribourg, \\ Switzerland \\ and}

Paul Scherrer Institute, 5232 Villingen PSI,

Switzerland

\section{G. Bison, and A. Weis}

Université de Fribourg, Chemin de Musée 3, 1700 Fribourg,

Switzerland
Number 3

May-June 2005
Key words: high precision magnetometry; laser spectroscopy; optical detected magnetic resonance; optical pumping. field fluctuations and gradients in a new experiment searching for a permanent electric dipole moment of the neutron. The intrinsic sensitivity of the device was determined to be $30 \mathrm{fT}$ in a measurement bandwidth of $1 \mathrm{~Hz}$, limited by laser noise In the shot noise limit the magnetometer can reach a sensitivity of $7 \mathrm{fT}$ for $1 \mathrm{~s}$ integration time. Test measurements of the stability of a $2 \mu \mathrm{T}$ magnetic field in a threefold magnetic shield have revealed fluctuations on the order of $200 \mathrm{fT}$ to $300 \mathrm{fT}$ with integration times in the range of $2 \mathrm{~s}$ to $100 \mathrm{~s}$. Those fluctuations were traced back to the stability of the power supply used to generate the magnetic field. The laser-pumped magnetometer fulfills the requirements set by the planned neutron electric dipole moment experiment.
Accepted: August 11, 2005

Available online: http://www.nist.gov/jres

\section{Introduction}

The precise measurement and control of magnetic fields and magnetic field fluctuations is an important step for experiments searching for a permanent electric dipole moment (EDM) of the neutron, and is one of the main factors limiting the accuracy. In a project approved by Paul Scherrer Institute (PSI) in Switzerland, a neutron EDM spectrometer is proposed [1] in which the neutron spin flip transition frequency is measured in four UCN storage chambers exposed to a homogenous $2 \mu \mathrm{T}$ magnetic field. In addition, each neutron chamber has two compartments in which the neutrons are exposed to a static electric field of $15 \mathrm{kV} / \mathrm{cm}$ oriented parallel/ antiparallel to the magnetic field. The signature of a finite EDM is a change of the neutron Larmor frequency synchronous with the rever sal of the relative orientations of the magnetic and elec- tric fields. This experiment imposes very stringent constraints on the homogeneity and stability of the magnetic field.

Fluctuations of the magnetic field will be monitored by a set of 16 optically-pumped alkali-vapor magnetometers (OPM), using the property that the oscillation frequency of an OPM is proportional to the modulus of the local magnetic field. The magnetometers considered for the first generation of the neutron EDM experiment are self-oscillating $\mathrm{Cs}$ vapor magnetometers (OPM) in the Mx configuration [2,3], optically pumped by spectral discharge lamps (LpOPM). However, it was previously shown that the replacement of the lamp by a resonant laser can lead to an appreciable gain in magnetometric sensitivity $[3,4]$. In that spirit we have designed and tested a laser-pumped OPM (LsOPM) with a geometry compatible with the neutron EDM experiment under construction. In case the laser version shows 
superior performance it might be considered as an alternative to the lamp-pumped magnetometers in the EDM experiment. Here we present the design and discuss the performance of Cs-LsOPMs operated in a phasestabilized mode.

\section{The Magnetometer Setup}

The magnetometer consists of three parts (Fig. 1): a) a sensor head containing no metallic parts except the r.f. coils, b) a base station mounted in a portable 19" rack drawer, which contains the frequency stabilized laser and all optics, and c) the frequency locking electronics. The sensor head is designed to fit into a tube of $104 \mathrm{~mm}$ diameter, coaxial with the $2 \mu \mathrm{T}$ field, and has a total length of $242 \mathrm{~mm}$. Its main component is a paraffin-coated, $7 \mathrm{~cm}$ diameter glass cell containing a droplet of cesium. The $\mathrm{Cs}$ atoms in the vapor are optically pumped by a circularly polarized light beam, oriented at $45^{\circ}$ to the magnetic field. An extended-cavity diode laser, which is stabilized on the $\mathrm{F}=4 \rightarrow \mathrm{F}=3$ hyperfine component of the $\mathrm{D}_{1}$ line at $894 \mathrm{~nm}$ wavelength by the dichroic atomic vapor laser lock (DAVLL) method [5], provides the resonant light which is guided from the base station to the sensor head via a $10 \mathrm{~m}$ long multimode fiber $(800 \mu \mathrm{m}$ core diameter). Note that a single diode laser can produce sufficient light power to drive all sensors in the experiment. The light transmitted through the cell is carried back to the detection unit by a similar fiber. The pumping process produces a polarization (magnetization) in the sample which undergoes a Larmor precession with the frequency $\omega_{\mathrm{L}}$. The precession is resonantly driven by a weak radio-frequency (rf) field (of amplitude $B_{1}$ and frequency $\omega_{\mathrm{rf}}$ ) applied with a pair of circular coils surrounding the cell. The absorption of the pumping light depends on the direction of the magnetization with respect to the light direction. Therefore the resonant precession frequency appears as an amplitude modulation on the light power transmitted through the cesium vapor. The detection of the modulated transmission by a photodetector allows a direct and real time measurement of the magnetic field in terms of the Larmor frequency.

The system behaves like a classical oscillator: when an $\mathrm{rf}$ field is applied, the phase of the response, i.e., the $\mathrm{AC}$ component of the transmitted laser intensity depends on the detuning $\delta \omega=\omega_{\mathrm{rf}}-\omega_{\mathrm{L}}$. For $\delta \omega<<0$ the atoms follow the driving field adiabatically and thus the radio frequency and the light modulation oscillate in phase. For $\delta \omega>>0$ the phase shift is $-180^{\circ}$, while on resonance $(\delta \omega=0)$ it becomes $-90^{\circ}$, while the modulation amplitude of the transmitted light reaches a maximum. In the phase-stabilized mode this dependency is used to lock the radio frequency to the Larmor frequency. The photodiode signal is analyzed by a dual-channel lock-in amplifier, referenced by the driving frequency of the rf coils produced by a voltage controlled oscillator (VCO). Either the phase or the dispersive in-phase output of the lock-in amplifier can be used in a feedback loop to lock the driving frequency to the Larmor frequency by stabilizing the phase shift to $-90^{\circ}$. The temporal response of

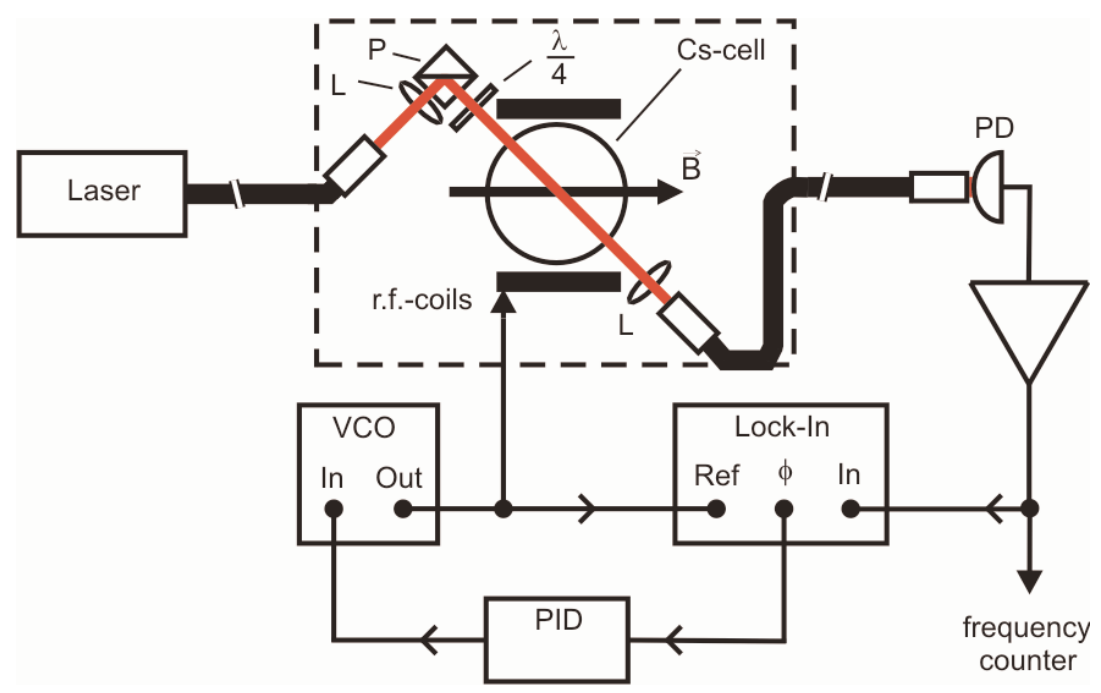

Fig. 1. Schematic setup of the phase-stabilized magnetometer. The dashed box indicates the sensor head. L: lens, P: polarizing beamsplitter, $\lambda / 4$ : quarter-wave plate, PD: photodiode, VCO: voltage-controlled oscillator, PID: feedback amplifier. The stabilization system of the laser frequency is not shown. 
the phase-stabilized magnetometer to field changes, i.e., its bandwidth is determined by the response time of the feedback loop and was measured to be in the order of $1 \mathrm{kHz}$.

\section{Performance of the Magnetometer}

\subsection{Intrinsic Magnetometric Sensitivity}

We define the intrinsic magnetometric sensitivity of the LsOPM in terms of the noise equivalent magnetic field (NEM). It is the square root of the power spectral density of magnetic field fluctuations at the magnetometer frequency. The field fluctuations produce a photodetector signal equal to the power spectral density of the photocurrent at the same frequency, each integrated over a bandwidth $f_{\mathrm{bw}}$. The NEM $\delta B$ is given by:

$$
\delta B=\frac{1}{\gamma} \cdot \frac{\Delta v_{\mathrm{HWHM}}}{S / N_{\mathrm{int}}},
$$

where $\gamma$ is a constant of approximately $3.5 \mathrm{~Hz} / \mathrm{nT}$ for ${ }^{133} \mathrm{Cs}$. The half width of the resonance is $\Delta v_{\text {ншнм }}$ and $S / N_{\text {int }}$ the signal-to-noise ratio of the photocurrent modulation in the phase-stabilized mode. The signal $S$ is the amplitude of the modulation. When measuring the intrinsic noise level, $N_{\text {int }}$, care has to be taken to eliminate external contributions, such as drifts and noise from external magnetic field sources. We therefore infer the intrinsic noise $N_{\text {int }}$ from a Fourier analysis of the photodiode signal when the magnetometer is operated under optimal conditions (Fig. 2). The central peak (the so-called carrier) is the oscillating magnetometer signal. It is superposed on a broad pedestal, which results mainly from a continuous distribution of sidebands due to imperfectly shielded low-frequency field fluctuations. The two discrete sidebands originate from interference of magnetic fields oscillating at the line frequency. The intrinsic noise level $N_{\text {int }}$ is given by the noise floor in Fig. 2, integrated over the detection bandwidth $f_{\mathrm{bw}}$. It lies $50 \%$ above the shot noise level $\Delta I=\sqrt{2 e I_{\mathrm{pc}} f_{\mathrm{bw}}}$ of the DC photocurrent $I_{\mathrm{pc}}$ in a bandwidth $f_{\text {bw }}$ of $1 \mathrm{~Hz}$.

The optimum operating point was found for a laser intensity of $9 \mu \mathrm{W} / \mathrm{mm}^{2}$ and a $B_{1}$ field amplitude of $2.7 \mathrm{nT}$, which yielded a linewidth $v_{\mathrm{HWHM}}=3.4(1) \mathrm{Hz}$ and a signal-to-noise ratio $S / N_{\text {int }}=97 \mathrm{~dB}=66000$. The corresponding intrinsic sensitivity is $\delta B_{\text {int }}=14.5 \mathrm{fT}$. If one assumes the noise to be white, the sensitivity scales with the square root of the bandwidth. Assuming the photodiode shot noise as ultimate sensitivity limit the LsOPM should reach a sensitivity of $\delta B_{\mathrm{SN}}=10 \mathrm{fT}$ in $1 \mathrm{~Hz}$ bandwidth, which corresponds to $\delta B_{\mathrm{SN}}=7 \mathrm{fT}$ for a $1 \mathrm{~s}$ integration time. The laser generated low frequency $1 / f$ noise is mixed to the Larmor frequency and thus contributes to the noise spectrum. It was found to exceed the white noise level $N_{\text {int }}$ by a factor 2 at the carrier frequency, which yields an intrinsic sensitivity of $\delta B^{*}{ }_{\text {int }}=29 \mathrm{fT}$. This contribution can be suppressed using an active stabilization of the laser power.

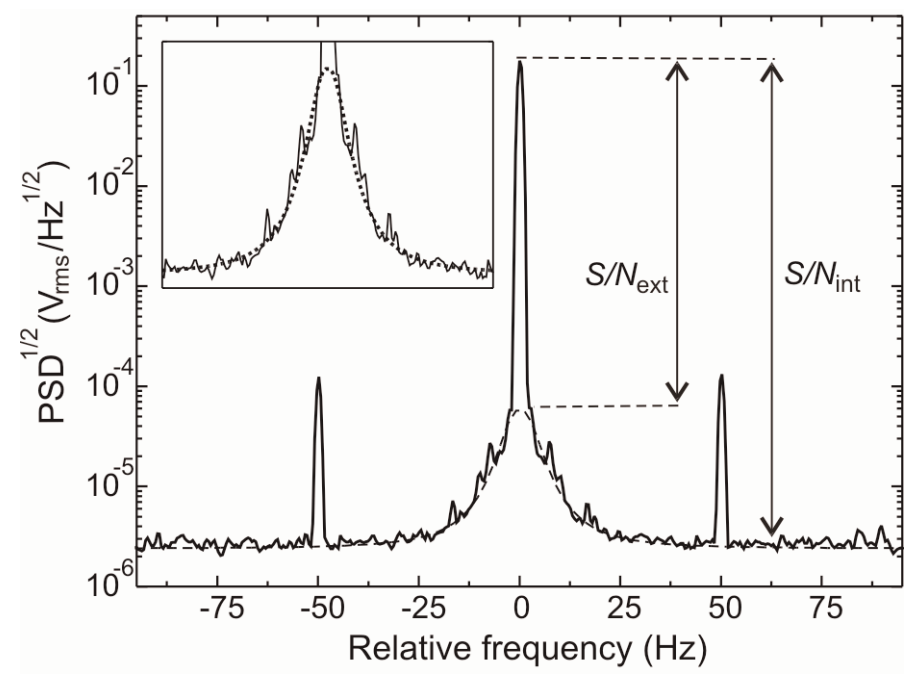

Fig. 2. Square root of the power spectral density (PSD) of the magnetometer output frequency relative to the Larmor frequency of $v_{\mathrm{L}}=7032 \mathrm{~Hz}$ (averaged 20 times). The spectrum was measured with a $1 \mathrm{~Hz}$ resolution bandwidth. The signal-to-noise ratio $S / N_{\text {int }}$ is approximately 66000 . The sidebands are due to imperfectly shielded magnetic field components oscillating at the $50 \mathrm{~Hz}$ powerline frequency. The dotted line is an approximation of the pedestal. The signal-tonoise ratio $S / N_{\text {ext }}$ due to external field noise is found to be approximately 2600 . 


\subsection{Application: Field Fluctuations in a Magnetic Shield}

We used the LsOPM to measure residual field fluctuations inside a three-layer magnetic shield. The Larmor frequency was recorded as multiple time series of several hours with a sampling rate of $0.1 \mathrm{~s}$. From each time series the Allan standard deviation [6] of the corresponding magnetic field inside the shield was calculated. A typical result is shown in Fig. 3. The observed fluctuations (curve a) are well above the intrinsic sensitivity level of the magnetometer (curves b). For integration times up to $1 \mathrm{~s}$ to $2 \mathrm{~s}$ the noise amplitude decreases as $\tau^{1 / 2}$, where $\tau$ is the integration time, indicating the presence of white field-amplitude noise. It can be characterized by a spectral amplitude of $413 \mathrm{fT} / \mathrm{Hz}^{1 / 2}$. With the estimated signal-to-noise ratio $S / N_{\text {ext }}=2600$ (cf. Fig. 2) we calculate from Eq. (1) a field stability of $370 \mathrm{fT}$ in a measurement bandwidth of $1 \mathrm{~Hz}$, which is in good agreement with the value in the Allan standard deviation plot. The minimum field fluctuations were found to be slightly larger than $200 \mathrm{fT}$ for an integration time of $4 \mathrm{~s}$. The central region of the Allan standard deviation plot shows a plateau for integration times of $2 \mathrm{~s}$ to $100 \mathrm{~s}$. It could be traced back to fluctuations of the $8 \mathrm{~mA}$ current producing the $2 \mu \mathrm{T}$ bias field and corresponds to a relative stability of $10^{-7}$ for the power supply. The Allan standard deviations for integration times exceeding $100 \mathrm{~s}$ are due to slow drifts of laboratory fields which are not completely suppressed by the three-layer shield, which has a measured longitudinal shielding factor of $10^{3}$. The influence of light shift effects [7] due to light intensity fluctuations are measured in an additional experiment. Figure $3 \mathrm{c}$ shows that those effects are negligible at the present level of field stability.

\section{Summary and Conclusion}

We have described the design and performance of a phase-stabilized cesium vapor magnetometer with a measurement bandwidth of $1 \mathrm{~Hz}$. The magnetometer has an intrinsic sensitivity of $29 \mathrm{fT}$, defined as the Allan standard deviation for a bandwidth of $1 \mathrm{~Hz}$. This value can be reduced by a factor of 2 if the $1 / f$ noise of the laser power can be lowered, e.g., by an active power stabilization. If the excess white noise can be reduced to the shot noise level a further increase of 1.5 can be obtained. Under these optimal conditions the LsOPM could reach a sensitivity of $7 \mathrm{fT}$ for an integration time of $1 \mathrm{~s}$. Field fluctuations of $200 \mathrm{fT}$ to $300 \mathrm{fT}$ were measured in a three-layer magnetic shield for integration times between $2 \mathrm{~s}$ and $100 \mathrm{~s}$. Light shift fluctuations, against which no particular precautions, except for the active frequency stabilization of the laser, were taken, are one to two orders of magnitude smaller than the residual field fluctuations in the shield. The laser-pumped OPM described here will thus be a valuable tool for fundamental physics experiments and compares very favorably with state-of-the-art lamppumped magnetometers as it will be demonstrated elsewhere.

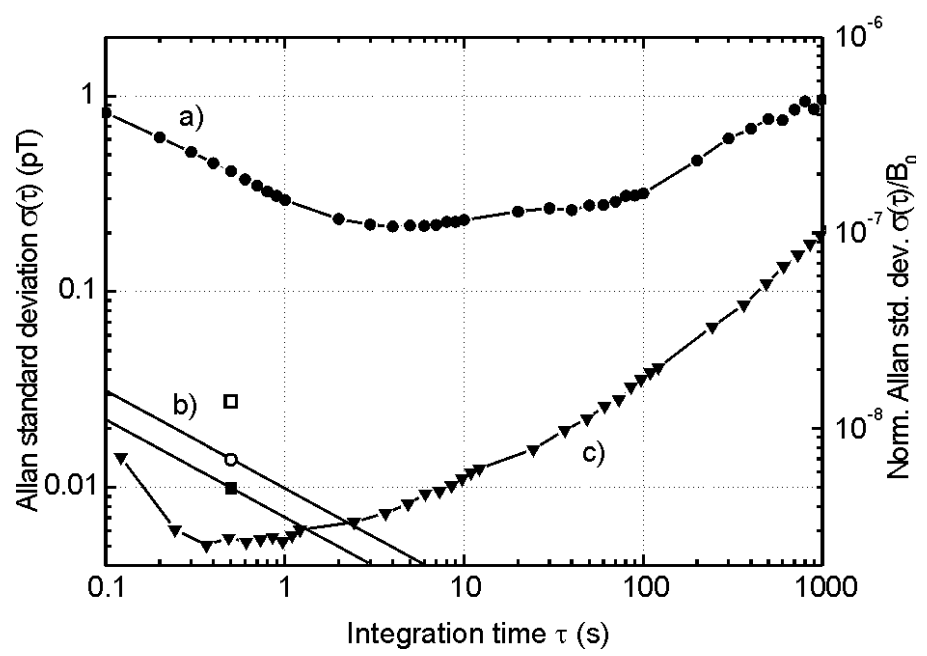

Fig. 3. a) Allan standard deviation of the magnetic flux density inside the magnetic shield. b) Intrinsic sensitivity limit. $\delta B^{*}{ }_{\text {int }}$ (square): sensitivity including $1 / f$ noise of the laser power; $\delta B_{\text {int }}$ (circle): sensitivity from experimentally determined white noise floor; $\delta B_{\mathrm{SN}}$ (filled square): anticipated sensitivity for shot noise limited operation. The slopes assume white noise. c) The triangles represent the light shift effect due to light power fluctuations. Solid lines in a) and c) are drawn to guide the eye. The dwell time of the frequency counter was $100 \mathrm{~ms}$. 


\section{Acknowledgements}

We are indebted to E. B. Aleksandrov, A. S. Pazgalev, and Robert Wynands for numerous fruitful discussions. We acknowledge financial support from Schweizerischer Nationalfonds, INTAS, and Paul Scherrer Institute (PSI). We thank PSI for the loan of the high-stability current source.

\section{References}

[1] E. B. Aleksandrov et al., A new precision measurement of the neutron electric dipole moment (EDM), PSI Proposal R-00$05.2(2002)$

[2] L. Bloom, Appl. Opt. 1, 61 (1962).

[3] E. B. Aleksandrov et al., Opt. Spectrosc. 78, 292 (1995).

[4] E. B. Aleksandrov et al., Sov. Phys. Tech. Phys. 35, 371 (1990).

[5] V. V. Yashchuk, D. Budker, and J. R. Davis, Rev. Sci. Instrum. 71, $341(2000)$.

[6] J. A. Barnes et al., IEEE Trans. Instrum. Meas. 20, 105 (1971).

[7] C. Cohen-Tannoudji, Ann. Phys. 7, 423 (1962); Ann. Phys. 7, 469 (1962).

About the authors: Antoine Weis is full professor at the Physics Department of the University of Fribourg and leader of the Fribourg Atomic Physics group (FRAP) at the Physics Department of the University of Fribourg. Stephan Groeger and Georg Bison are Ph.D. students in the FRAP group. 